\title{
Short-term changes in liver tests predict long-term mortality
}

\author{
Eliana Saffouri, ${ }^{1}$ Eugénie S Lim, ${ }^{2}$ Susan W Kim, ${ }^{3}$ Paul Hakendorf, ${ }^{4}$ \\ Campbell H Thompson ${ }^{2}$
}

\begin{abstract}
- Additional material is published online only. To view please visit the journal online (http://dx.doi.org/10.1136/ flgastro-2015-100669).

1 Department of Gastroenterology, Forth Valley Royal Hospital, NHS Forth Valley, Lambert, Scotland, UK ${ }^{2}$ Department of Medicine University of Adelaide, Adelaide, South Australia, Australia

${ }^{3}$ Flinders Centre for Epidemiology and Biostatistics, Flinders University, Adelaide, South Australia, Australia ${ }^{4}$ Flinders' Southern Adelaide Clinical School, Flinders University, Adelaide, South Australia, Australia
\end{abstract}

Correspondence to Dr Eugénie S Lim, Department of Medicine, University of Adelaide, Level 1, Eleanor Harrald Building, Frome Road Adelaide, SA 5005, Australia; eugenie.lim@gmail.com

Received 1 December 2015 Revised 23 January 2016 Accepted 7 February 2016 Published Online First 8 March 2016

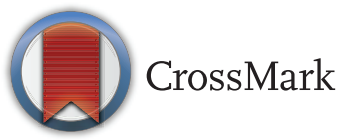

\begin{tabular}{l}
\hline To cite: Saffouri E, Lim ES, \\
Kim SW, et al. Frontline \\
Gastroenterology \\
2016:7:234-239.
\end{tabular}

To cite: Saffouri E, Lim ES, 2016:7:234-239.

\begin{abstract}
Objective To determine whether short-term changes in liver tests (bilirubin, albumin, gamma glutamyl transferase, alkaline phosphatase, alanine aminotransferase (ALT) and aspartate aminotransferase) predict 12-month mortality and, if so, which test is most informative.

Design Retrospective review of general medicine inpatients at a tertiary hospital (2005-2012) identified non-elective admissions of minimum 7 days' duration. Patients with liver disease, malignancy, admission to the intensive care unit or inpatient mortality were excluded. Linear spline modelled the vector of intraadmission change from admission. The association between 12-month mortality and admission and intra-admission changes in liver tests was assessed by logistic regression modelling, adjusted for age, gender, comorbidity index and heart failure.
\end{abstract}

Results 12 -month mortality was $17 \%$ in 4160 patients analysed. 12-month mortality for patients with abnormally low albumin at admission was $5 \%$ higher per $1 \mathrm{~g} / \mathrm{L}$ below $34 \mathrm{~g} / \mathrm{L}$ (OR 0.95, 95\% Cl 0.93 to 0.98, $\mathrm{p}<0.001)$. Albumin and ALT were the only tests for which an intra-admission change significantly predicted mortality; the predictive effects were additive. 12-month mortality was greater by $4 \%$ per $1 \mathrm{~g} / \mathrm{L}$ intra-admission decrement in albumin (OR 1.04, 95\% Cl 1.02 to $1.06, p<0.001)$ and $6 \%$ per $100 \mathrm{IU} / \mathrm{L}$ intraadmission increment in ALT (OR 1.06, 95\% Cl 1.02 to $1.1, p=0.005)$. Intra-admission changes were superior to admission values in predicting mortality.

Conclusions Changes in liver tests predict longterm mortality better than a single value and provide prognostic information more quickly than long-term monitoring. In the absence of known liver disease, albumin predicts long-term mortality better than transaminases. The patient whose albumin decreases in the short term is at high risk of death within 1 year, even from a normal baseline.

\section{BACKGROUND}

The liver can be assessed indirectly by performing certain blood tests, namely albumin, bilirubin, alkaline phosphatase (ALP), gamma glutamyl transferase (GGT), alanine aminotransferase (ALT) and aspartate aminotransferase (AST). These tests can also reflect non-hepatic pathology such as haemolysis in the case of bilirubin or, in the case of albumin, poor nutrition or protein loss from the gut or kidney. Liver dysfunction can prompt and influence diagnostic and therapeutic processes but can also act as a prognostic tool. Abnormal liver tests are indicative of a higher risk of short-term and long-term mortality, often from cardiovascular disease or heart failure. ${ }^{1-5}$ High bilirubin, GGT, ALT and AST at study baseline have been associated with long-term mortality in outpatients with chronic heart failure, ${ }^{1}{ }^{3}$ while other studies demonstrate that an increase in $\mathrm{GGT}^{4}$ or a reduction in albumin ${ }^{6}$ over a number of years is independently associated with an increased risk of cardiovascular disease and, in the case of GGT at least, long-term mortality. The implications for mortality of short-term changes in liver tests have not been demonstrated.

Heart failure and chronic liver disease are common comorbidities that will have an impact on 12-month mortality and also increase the variability of liver tests. ${ }^{1}{ }^{7-9}$ Thus, these conditions should be accounted for when describing associations between liver tests and mortality. The general medicine inpatient population is typically older subjects with complex comorbidities, including admission diagnoses of acute myocardial infarc- 
tion, cerebrovascular disease, dementia, chronic obstructive lung disease, chronic heart failure and/or metastatic cancer. ${ }^{10}$ The mortality in the year after discharge of this group nears $20 \% .{ }^{11}$ Identification of those at greatest risk of death would assist planning further management and clarifying wishes for end-of-life care. The aim of this study was to characterise the prognostic utility of measuring changes in liver tests during an admission by applying a mortality prediction model that recognised the confounding effects upon liver test variability of chronic heart and liver diseases, both of which are common in general medicine inpatients.

\section{METHODS}

Retrospective review of data for inpatients at a tertiary hospital from 2005 to 2012 identified patients non-electively admitted to general medicine with a 7-day minimum length of stay and with liver tests (albumin, bilirubin, ALP, GGT, ALT, AST) measured upon admission and on at least one other day during the first admission of the study period. Patients admitted to general medicine were typically those with multisystem comorbidities that would be managed best by a general physician and an admission diagnosis that did not require the care of a subspecialty unit, such as cardiac monitoring or procedural intervention. Patient exclusion criteria were an existing coded diagnosis of liver disease or any malignancy, an admission to the intensive care unit (ICU) or death during admission.

The Charlson Index (CI) was calculated for each patient with a Stata user-written routine for the 26 primary and secondary diagnoses from the hospital administrative database, coded by International Classification of Diseases (ICD)-10. ${ }^{2}$ CIs were then categorised as 0,1 or $\geq 2$ to control for the effect of no, 1 or $\geq 2$ comorbidities, respectively. Specific note was made of patients with a coded diagnosis of heart failure.

Mortality at 3 months and 12 months was determined from a mandatory registry maintained by the Births, Deaths and Marriages Registration Office of the Government of South Australia (http://www.cbs.sa. gov.au/births-deaths-marriages/). It was assumed that all patients remained within this jurisdiction for 12 months following discharge from hospital.

Liver tests were measured at the laboratories of SA Pathology (Roche Diagnostics, Sydney, Australia), with reference intervals as follows: albumin $34-48 \mathrm{~g} / \mathrm{L}$, bilirubin 2-24 $\mu \mathrm{mol} / \mathrm{L}$, ALP 30-110 U/L, GGT $\leq 60 \mathrm{U} / \mathrm{L}$, ALT $\leq 55 \mathrm{U} / \mathrm{L}$ and AST $\leq 45 \mathrm{U} / \mathrm{L}$. All statistical analyses were performed with Stata V.13.1 (StataCorp, USA).

Cohort characteristics were examined using the $\chi^{2}$ test for categorical variables and the Mann-Whitney test for continuous variables as they were mostly not normally distributed. Piecewise logistic regression modelling was used to examine the effects of liver tests upon 3-month and 12-month mortality. A prediction model of 12-month mortality, adjusted for age, gender, heart failure and CI was constructed (Model 1), to which liver tests measured at admission were added (Model 2), followed by intra-admission changes in those tests (Model 3). Liver tests measured at admission were modelled as linear spline with nodes corresponding to the reference range of each test result. Intra-admission changes were also modelled using linear spline but with the node at 0 to characterise either an increment or a decrement in liver tests. Linear splines allowed the effect of liver tests measured at admission to have a different slope for normal and abnormal test results and the effect of the intra-admission changes to have a different slope for positive and negative changes. Summary of statistics is displayed as number (\%) for categorical variables and median with IQR (lower to upper quartile) for continuous variables. P-values less than 0.05 were considered statistically significant.

\section{RESULTS}

There were 6011 patients admitted to general medicine without any ICU management and of those, 5061 had all six liver tests measured both upon admission and on at least one other day during their first admission within the study period. Two hundred and fifty-seven patients died during their admission and a further 644 patients were excluded for a preexisting diagnosis of malignancy or liver disease. Patient characteristics at baseline of the final 4160

\section{Table 1 Characteristics of cohort on admission}

\begin{tabular}{lc}
\hline & Study cohort \\
\hline Number & 4160 \\
Age (years) & $79(66,86)$ \\
Gender & \\
Male & $1941(46.7 \%)$ \\
Female & $2219(53.3 \%)$ \\
Charlson Index & \\
0 & $1467(35.3 \%)$ \\
1 & $1199(28.8 \%)$ \\
$\geq 2$ & $1494(35.9 \%)$ \\
Diagnosis of heart failure & $1035(24.9 \%)$ \\
Length of admission (days) & $11.8(8.9,17.3)$ \\
Deceased at 30 days & $189(4.5 \%)$ \\
Deceased at 12 months & $716(17.2 \%)$ \\
Liver tests on admission & \\
Albumin (g/L) & $35(31,38)$ \\
Bilirubin ( $\mu$ mol/L) & $10(6,14)$ \\
ALP (U/L) & $88(69,116)$ \\
GGT (U/L) & $41(24,84)$ \\
ALT (U/L) & $21(14,35)$ \\
AST (U/L) & $31(23,46)$ \\
\hline Data are presented as median (IQR) or num (percenta &
\end{tabular}

Data are presented as median (IQR) or number (percentage).

ALP, alkaline phosphatase; ALT, alanine aminotransferase; AST, aspartate aminotransferase; GGT, gamma glutamyl transferase. 
patients remaining for analysis are shown in table 1 . The mean age of patients was 74 years. Seven hundred and sixteen patients $(17.6 \%)$ were deceased at 12 months, including $25 \%$ of those patients admitted with a $\mathrm{CI} \geq 2$. A diagnosis of heart failure was present in $25 \%$ of the cohort; $27 \%$ of these patients died within 12 months compared with $14 \%$ of those without a diagnosis of heart failure $(p<0.001)$.

The majority of patients had liver tests in the normal range on admission, although nearly 40\% were hypoalbuminaemic (see online supplementary table S1). Twenty per cent of these patients with a low albumin at admission were deceased at 12 months, which is a significantly higher proportion of deaths than observed among those with a normal or high albumin at admission $(p<0.001)$. No difference in mortality was observed for patients with any one liver test high on admission compared with those with normal tests on admission. Intra-admission changes in liver tests were small (see online supplementary table S2), noting that the median length of admission was less than 12 days. Fewer than $25 \%$ of patients had an ALT or AST increment to achieve intra-admission levels twice the upper limit of normal. The majority of patients $(77 \%)$ had a predominant fall in albumin during their admission and a 12-month mortality of $18 \%$, compared with $14.6 \%$ mortality in those whose albumin did not decrement during admission $(\mathrm{p}=0.017)$.

Table 2 shows the effects of liver tests at admission and intra-admission changes in those tests upon 12-month mortality. Albumin was the only test for which admission levels significantly predicted mortality (table 2). Model 2 demonstrated that 12-month mortality for patients with abnormally low albumin at admission was $5 \%$ higher for every $1 \mathrm{~g} / \mathrm{L}$ below $34 \mathrm{~g} /$ $\mathrm{L}(\mathrm{p}<0.001)$ and that even for patients whose admission albumin was in the normal range, 12-month mortality increased by $3 \%$ for every $1 \mathrm{~g} / \mathrm{L}$ below the upper limit of normal $(p=0.035)$. Albumin and ALT were the only liver tests for which an intra-admission change significantly predicted mortality, and the predictive effects were additive (Model 3). 12-month mortality increased by $4 \%$ for every $1 \mathrm{~g} / \mathrm{L}$ intra-admission decrement in albumin $(\mathrm{p}<0.001)$; mortality reduction in patients whose albumin incremented during their admission approached statistical significance $(p=0.051)$. 12-month mortality was greater by $6 \%(\mathrm{p}=0.005)$ per $100 \mathrm{U} / \mathrm{L}$ intra-admission increment in ALT, regardless of ALT at admission. This impact of admission albumin values and intra-admission changes in albumin and ALT on mortality was seen as early as 30 days (see online supplementary table S3).

The utility of these data in prognostication was further illustrated by graphing mortality against intra-admission changes in albumin and ALT for a patient with mean values in this cohort: that is, a 74-year-old man with CI of 1 , no heart failure and albumin at admission of $34.5 \mathrm{~g} / \mathrm{L}$. An albumin decrement was more detrimental to prognosis than an equivalent increment was beneficial (figure 1); the

Table 2 Effects of liver tests taken at admission and intra-admission changes in liver tests upon 12-month mortality

\begin{tabular}{|c|c|c|c|c|c|c|}
\hline \multirow[b]{2}{*}{ Deceased at 12 months } & \multicolumn{2}{|l|}{$\begin{array}{l}\text { Model 1: } \\
\text { Without liver tests }\end{array}$} & \multicolumn{2}{|c|}{$\begin{array}{l}\text { Model 2: } \\
\text { With admission liver tests }\end{array}$} & \multicolumn{2}{|c|}{$\begin{array}{l}\text { Model 3: } \\
\text { With intra-admission changes } \\
\text { in liver tests }\end{array}$} \\
\hline & OR $(95 \% \mathrm{Cl})$ & p Value & OR $(95 \% \mathrm{Cl})$ & p Value & OR $(95 \% \mathrm{Cl})$ & $\mathrm{p}$ Value \\
\hline$\overline{\text { Age }}$ & $1.06(1.05$ to 1.06$)$ & $<0.001$ & 1.06 (1.05 to 1.07$)$ & $<0.001$ & $1.06(1.05$ to 1.07$)$ & $<0.001$ \\
\hline Female & $0.74(0.63$ to 0.88$)$ & 0.001 & $0.74(0.62$ to 0.87$)$ & $<0.001$ & $0.74(0.62$ to 0.88$)$ & 0.001 \\
\hline \multicolumn{7}{|l|}{ Charlson Index } \\
\hline 0 & 1 & & 1 & & 1 & \\
\hline 1 & $1.47(1.15$ to 1.87$)$ & $<0.001$ & 1.47 (1.15 to 1.87$)$ & $<0.001$ & $1.48(1.16$ to 1.89$)$ & $<0.001$ \\
\hline$\geq 2$ & 1.91 (1.5 to 2.44$)$ & & $1.93(1.51$ to 2.46$)$ & & $1.88(1.47$ to 2.41$)$ & \\
\hline Heart failure & $1.41(1.15$ to 1.71$)$ & 0.001 & $1.38(1.13$ to 1.68$)$ & 0.002 & $1.37(1.12$ to 1.67$)$ & 0.002 \\
\hline \multicolumn{7}{|c|}{ Effect of baseline albumin (per $1 \mathrm{~g} / \mathrm{L}$ ) } \\
\hline Baseline $<34 \mathrm{~g} / \mathrm{L}$ & & & $0.95(0.93$ to 0.98$)$ & 0.001 & $0.93(0.9$ to 0.96$)$ & $<0.001$ \\
\hline Baseline 34-48 g/L & & & $0.97(0.94$ to 1$)$ & 0.035 & $0.95(0.92$ to 0.98$)$ & 0.003 \\
\hline Baseline >48 g/L & & & $0.8(0.25$ to 2.53$)$ & 0.703 & $0.75(0.23$ to 2.46$)$ & 0.632 \\
\hline \multicolumn{7}{|c|}{ Effect of intra-admission change in albumin (per $1 \mathrm{~g} / \mathrm{L}$ change) } \\
\hline Decrement & & & & & $1.04(1.02$ to 1.06$)$ & $<0.001$ \\
\hline Increment & & & & & $0.96(0.92$ to 1$)$ & 0.051 \\
\hline \multicolumn{7}{|c|}{ Effect of intra-admission change in ALT (per $100 \mathrm{U} / \mathrm{L}$ change) } \\
\hline Decrement & & & & & $1.01(0.96$ to 1.06$)$ & 0.624 \\
\hline Increment & & & & & $1.06(1.02$ to 1.1$)$ & 0.005 \\
\hline
\end{tabular}

ALT, alanine aminotransferase. 


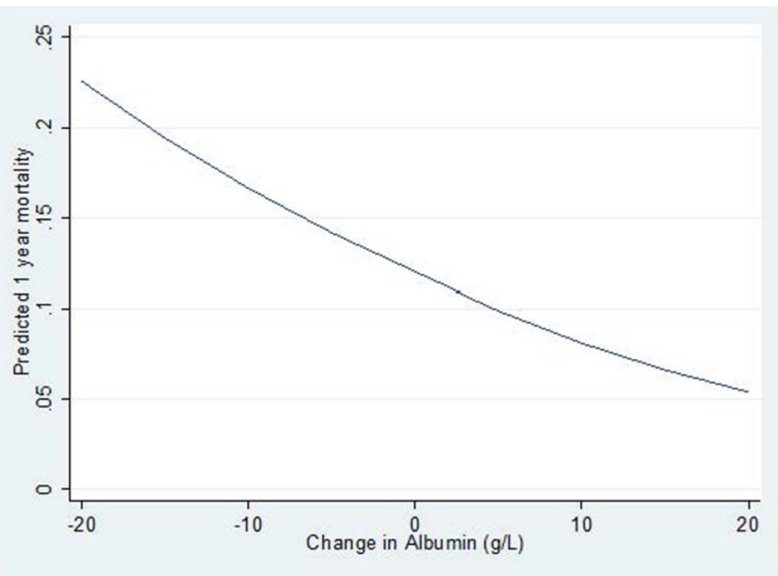

Figure 1 Predicted mortality for intra-admission change in albumin, calculated for a 74-year-old man with Charlson Index of 1 , no heart failure, admission albumin of $34.5 \mathrm{~g} / \mathrm{L}$ and no intra-admission change in alanine aminotransferase.

converse was true for ALT (figure 2). Figure 3 displays mortality curves for the average patient with four potential albumin values at admission and no intra-admission change in ALT. These curves demonstrate the additive value of assessing both albumin at admission and its intra-admission change, because it is apparent that albumin decrements from below normal baseline values had a greater impact on mortality than equivalent decrements from within the normal range.

\section{DISCUSSION}

This study has confirmed that some of the previously described associations between deranged liver tests and mortality apply to the elderly general medicine population, irrespective of known heart failure, and deliberately excluding any patient with chronic liver disease or malignancy. This cohort of inpatients demonstrated a one in six chance of dying within 12 months of discharge. While this likely reflects the

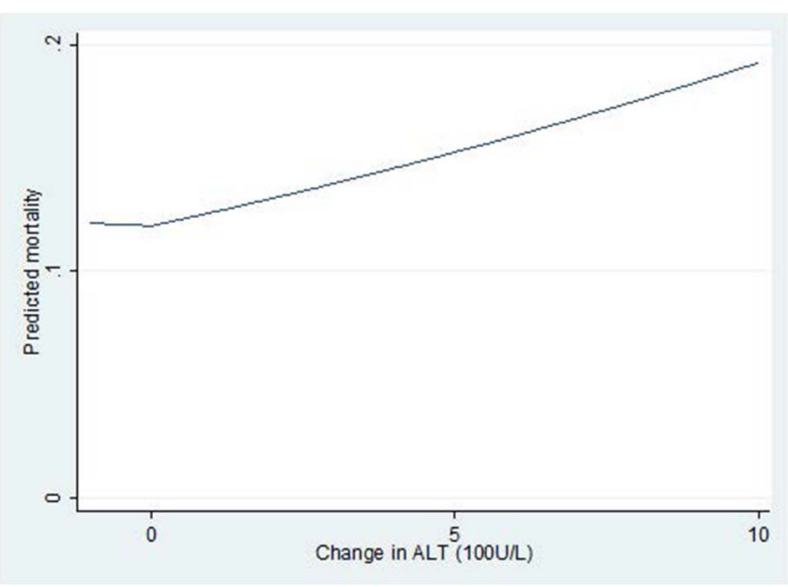

Figure 2 Predicted mortality for intra-admission change in alanine aminotransferase (ALT), calculated for a 74-year-old man with Charlson Index of 1, no heart failure, admission albumin of $34.5 \mathrm{~g} / \mathrm{L}$ and no intra-admission change in albumin.

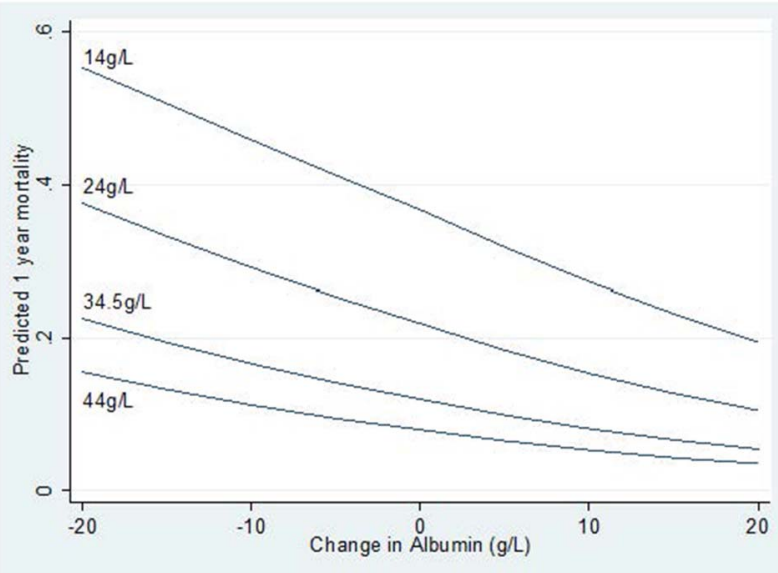

Figure 3 Predicted mortality for intra-admission change in albumin for four different admission values of albumin, calculated for a 74-year-old man with Charlson Index of 1, no heart failure and no intra-admission change in alanine aminotransferase.

age and accompanying comorbidities of the population, short-term changes in albumin and ALT were identified as prognostic markers.

Of all liver tests, albumin was the most sensitive marker of 12-month mortality. This was true of a single measurement as previously demonstrated ${ }^{3} 1314$ and of an albumin trend. Importantly, the likelihood of mortality was predicted by the extent of a decrement in albumin over a number of days. Serum levels of albumin reflect liver synthetic function but it is well established that they are also mediated by systemic (ie, non-hepatic) pathophysiology. ${ }^{15}$ Critical illness may cause albumin levels to drop by altering the distribution of albumin between intravascular and extravascular compartments, largely due to an increase in capillary leakage. ${ }^{15} 16$ Patients managed in the ICU were excluded from our study; future work could exclude even those patients with the systemic inflammatory response syndrome. While a proportion of the critically ill may have been deemed ineligible for ICU and therefore failed exclusion from this analysis, patients who were declined intensive support yet survived admission were likely few. While albumin has traditionally been viewed as a biomarker of nutritional status, ${ }^{17}$ the evidence for this is controversial and nutritional interventions have not definitively been shown to sustain normoalbuminaemia or improve mortality. ${ }^{18}$ Since attempts to correct hypoalbuminaemia per se are ineffective, ${ }^{16}{ }^{18}$ it is only by managing underlying disease that patient morbidity and mortality might be reduced. This study has therefore highlighted that the presence of abnormalities in a patient's serum albumin measurement-either a single low level or, more significantly, a decrement even within the normal range-must prompt evaluation for an underlying cause.

Changes in hepatic enzymes were much less sensitive than changes in albumin for the prediction of 
long-term mortality. Transaminases, rather than ALP and GGT, performed best. In this population, with median admission albumin $35 \mathrm{~g} / \mathrm{L}$ and ALT $21 \mathrm{U} / \mathrm{L}$, an equivalent increase in 12-month mortality was predicted for $<5 \%$ change in albumin and $>300 \%$ change in ALT. Still, the recognition of these changes can assist the identification of those at risk of death within 12 months. Importantly, small changes in albumin, even within the normal range, may confer measurable risk; conversely, substantial changes in ALT may be required for serious effect. Future work must study the clinical consequences of targeted interventions for this vulnerable population.

In contrast to previous studies, ${ }^{4}{ }^{19-21}$ no association with 12-month mortality was found for abnormalities in any of bilirubin, GGT, ALP or AST. That we did not identify mortality risk with all liver tests may reflect exclusion of patients with established liver disease and the adjustment of all associations for the coded diagnosis of heart failure. Previously recognised associations are presumably explained by heart failure and liver disease. The persistence and strength of the association of decrements in albumin with mortality after taking these conditions into account possibly reflect non-hepatic influences on serum albumin, such as inflammation and, possibly, nutrition. Deranged transaminases, reflective of hepatocellular insult, are perhaps less susceptible than albumin to systemic illness and do not portend a significant mortality risk when elevation is mild. The combination of deranged albumin and ALT increased mortality, suggesting that hypoalbuminaemia of evolving liver disease is more lethal than an equivalent decrement in albumin seen in systemic inflammation or malnutrition. Whatever its aetiology, early evaluation of the elderly patient with decrementing albumin is warranted.

This study is limited as a single-centre, retrospective, observational analysis, although this is somewhat offset by its cohort of over 4000 patients. The data set lacks coagulation studies and the categorisation of cause of death. The use of linear spline to model the effects of admission liver tests and intra-admission changes in liver tests on mortality could oversimplify trends; however, the linearity assumption was checked and satisfied for all variables included in the model. The statistical significance of the effect size for those with an admission albumin greater than $48 \mathrm{~g} / \mathrm{L}$ was not achieved but, with only 19 patients in this category, lack of power is thought likely.

Beyond the identification of long-term mortality risks such as malignancy, heart failure and liver disease, clinicians should be prompted by ambient albumin levels and any short-term deterioration in albumin or ALT to begin long-term planning of management for the older general medicine inpatient. Recognition of these particular liver tests and the magnitude of their change as markers of mortality must trigger clinicians to evaluate more traditional risk factors for mortality, plan timely outpatient review and initiate discussions regarding end-of-life care.

\section{Key messages}

What is already known on this topic?

- Elevated levels of bilirubin and transaminases in patients with chronic heart failure are associated with an increased risk of cardiovascular disease and long-term mortality.

- A decline in serum albumin over a number of years is associated non-specifically with increased mortality.

\section{What does this study add?}

- Associations between derangement in liver tests and mortality are mostly explained by the presence of established heart failure or liver disease.

> In the absence of recognised chronic heart and liver diseases, short-term changes in liver tests, over a number of days, can be informative for 12-month prognostication and are more useful than a single measurement.

- Short-term small decrements in albumin, even within the laboratory reference interval, anticipate higher 12-month mortality.

- Substantial elevations in alanine aminotransferase (ALT) are required to confer an increased risk of death at 12 months.

\section{How might it impact on clinical practice in the} foreseeable future?

- The measurement of a change in liver tests over a number of days is more useful than a single reading and more practical and timely than monitoring over several months.

- Short-term changes in liver tests can prompt an evaluation for underlying systemic inflammatory disorders, malnutrition, undiagnosed malignancy, liver disease or heart failure.

- Recognition of deteriorating albumin or ALT (but particularly both) can help to select patients for early follow-up or end-of-life care planning.

Contributors ES and ESL: study conception and design; interpretation of data; manuscript preparation. SWK: study conception and design; data acquisition; data analysis; manuscript preparation. $\mathrm{PH}$ : data acquisition; data analysis. CHT: study conception and design; data acquisition; data analysis; interpretation of data; manuscript preparation.

Competing interests None declared.

Provenance and peer review Not commissioned; externally peer reviewed. 


\section{REFERENCES}

1 Batin P, Wickens M, McEntegart D, et al. The importance of abnormalities of liver function tests in predicting mortality in chronic heart failure. Eur Heart J 1995;16:1613-18.

2 van Deursen VM, Edwards C, Cotter G, et al. Liver function, in-hospital, and post-discharge clinical outcome in patients with acute heart failure-results from the relaxin for the treatment of patients with acute heart failure study. J Card Fail 2014;20:407-13.

3 Allen LA, Felker GM, Pocock S, et al. Liver function abnormalities and outcome in patients with chronic heart failure: data from the Candesartan in Heart Failure: Assessment of Reduction in Mortality and Morbidity (CHARM) program. Eur J Heart Fail 2009;11:170-7.

4 Strasak AM, Kelleher CC, Klenk J, et al. Longitudinal change in serum gamma-glutamyltransferase and cardiovascular disease mortality: a prospective population-based study in 76,113 Austrian adults. Arterioscler Thromb Vasc Biol 2008;28:1857-65.

5 Djoussé L, Rothman KL, Cupples LA, et al. Serum albumin and risk of myocardial infarction and all-cause mortality in the Framingham Offspring Study. Circulation 2002;106: 2919-24.

6 Schalk BWM, Visser M, Bremmer MA, et al. Change of serum albumin and risk of cardiovascular disease and all-cause mortality: longitudinal aging study Amsterdam. Am J Epidemiol 2006;164:969-77.

7 Kubo SH, Walter BA, John DA, et al. Liver function abnormalities in chronic heart failure. Influence of systemic hemodynamics. Arch Intern Med 1987;147:1227-30.

8 Said A, Williams J, Holden J, et al. Model for end stage liver disease score predicts mortality across a broad spectrum of liver disease. J Hepatol 2004;40:897-903.

9 Pratt DS, Kaplan MM. Evaluation of abnormal liver-enzyme results in asymptomatic patients. N Engl J Med 2000;342:1266-71.
10 Russell P, Hakendorf P, Thompson C. Inter-hospital lateral transfer does not increase length of stay. Aust Health Rev 2015;39:400-3.

11 Li JY, Yong TY, Hakendorf P, et al. The survival of patients with not-for-resuscitation orders. QJM 2013;106:903-7.

12 Quan H, Li B, Couris CM, et al. Updating and validating the Charlson comorbidity index and score for risk adjustment in hospital discharge abstracts using data from 6 countries. Am J Epidemiol 2011;173:676-82.

13 Jellinge ME, Henriksen DP, Hallas P, et al. Hypoalbuminemia is a strong predictor of 30-day all-cause mortality in acutely admitted medical patients: a prospective, observational, cohort study. PloS ONE 2014;9:e105983.

14 Hannan JL, Radwany SM, Albanese T. In-hospital mortality in patients older than 60 years with very low albumin levels. J Pain Symptom Manage 2012;43:631-7.

15 Rothschild MA, Oratz M, Schreiber SS. Albumin metabolism. Gastroenterology 1973;64:324-37.

16 Nicholson JP, Wolmarans MR, Park GR. The role of albumin in critical illness. Br J Anaesth 2000;85:599-610.

17 McWhirter JP, Pennington CR. Incidence and recognition of malnutrition in hospital. BMJ 1994;308:945-8.

18 Friedman AN, Fadem SZ. Reassessment of albumin as a nutritional marker in kidney disease. J Am Soc Nephrol 2010;21:223-30.

19 Fulks M, Stout RL, Dolan VF. Using liver enzymes as screening tests to predict mortality risk. J Insur Med 2008;40:191-203.

20 Ong KL, Allison MA, Cheung BMY, et al. The relationship between total bilirubin levels and total mortality in older adults: the United States National Health and Nutrition Examination Survey (NHANES) 1999-2004. PLoS ONE 2014;9:e94479.

21 López-Velázquez JA, Chávez-Tapia NC, Ponciano-Rodríguez G, et al. Bilirubin alone as a biomarker for short-term mortality in acute-on-chronic liver failure: an important prognostic indicator. Ann Hepatol 2013-2014;13:98-104. 